Supplement of

\title{
An in situ gas chromatograph with automatic detector switching between PTTR-and EI-TOF-MS: isomer-resolved measurements of indoor air
}

Megan S. Claflin et al.

Correspondence to: Megan S. Claflin (mclaflin@ aerodyne.com)

The copyright of individual parts of the supplement might differ from the CC BY 4.0 License. 

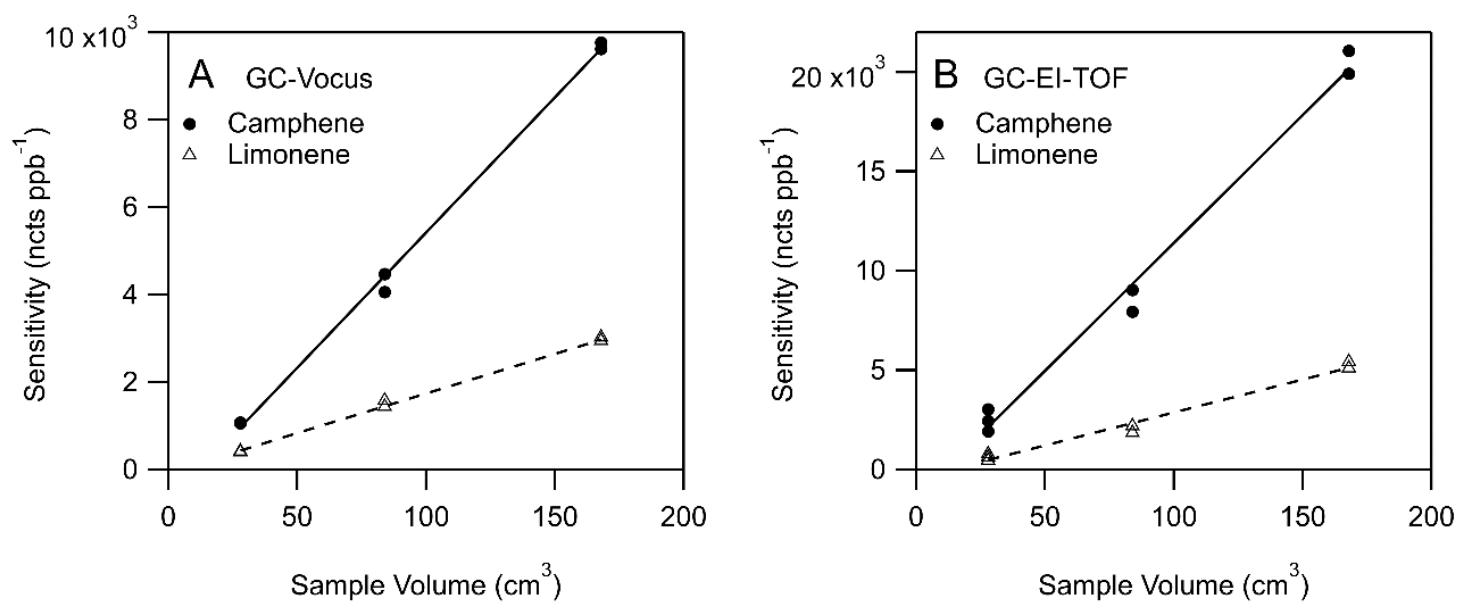

Figure S1. Monoterpene sensitivities (normalized counts per ppb) versus sample volume for limonene and camphene acquired by A) GC-Vocus and B) GC-EI-TOF. Monoterpenes were quantified using their protonated molecular ion $\left(\mathrm{C}_{10} \mathrm{H}_{17}{ }^{+}\right)$for GC-Vocus, and characteristic fragment ion $\left(\mathrm{C}_{7} \mathrm{H}_{9}{ }^{+}\right)$for GC-EITOF. 
Table S1. Sensitivity uncertainties (1- $\sigma)$ for the GC-EI-TOF and GC-Vocus calibrations.

\begin{tabular}{|ccc|}
\hline & \multicolumn{2}{c|}{ Sensitivity Uncertainty (\%) } \\
\cline { 2 - 3 } a-pinene & GC-EI-TOF & GC-Vocus \\
\hline camphene & $+/-14.5$ & $+/-5.7$ \\
B-pinene & $+/-8.5$ & $+/-5.4$ \\
carene & $+/-13.4$ & $+/-6.1$ \\
limonene & $+/-14.7$ & $+/-4.4$ \\
benzene & $+/-11.2$ & $+/-5.4$ \\
toluene & $+/-11.8$ & $+/-3.8$ \\
ethyl-benzene & $+/-8.4$ & $+/-6.2$ \\
m\&p-xylenes & $+/-11.5$ & $+/-5.2$ \\
o-xylene & $+/-12.4$ & $+/-6.1$ \\
& $+/-12.3$ & +-3.9 \\
\hline
\end{tabular}



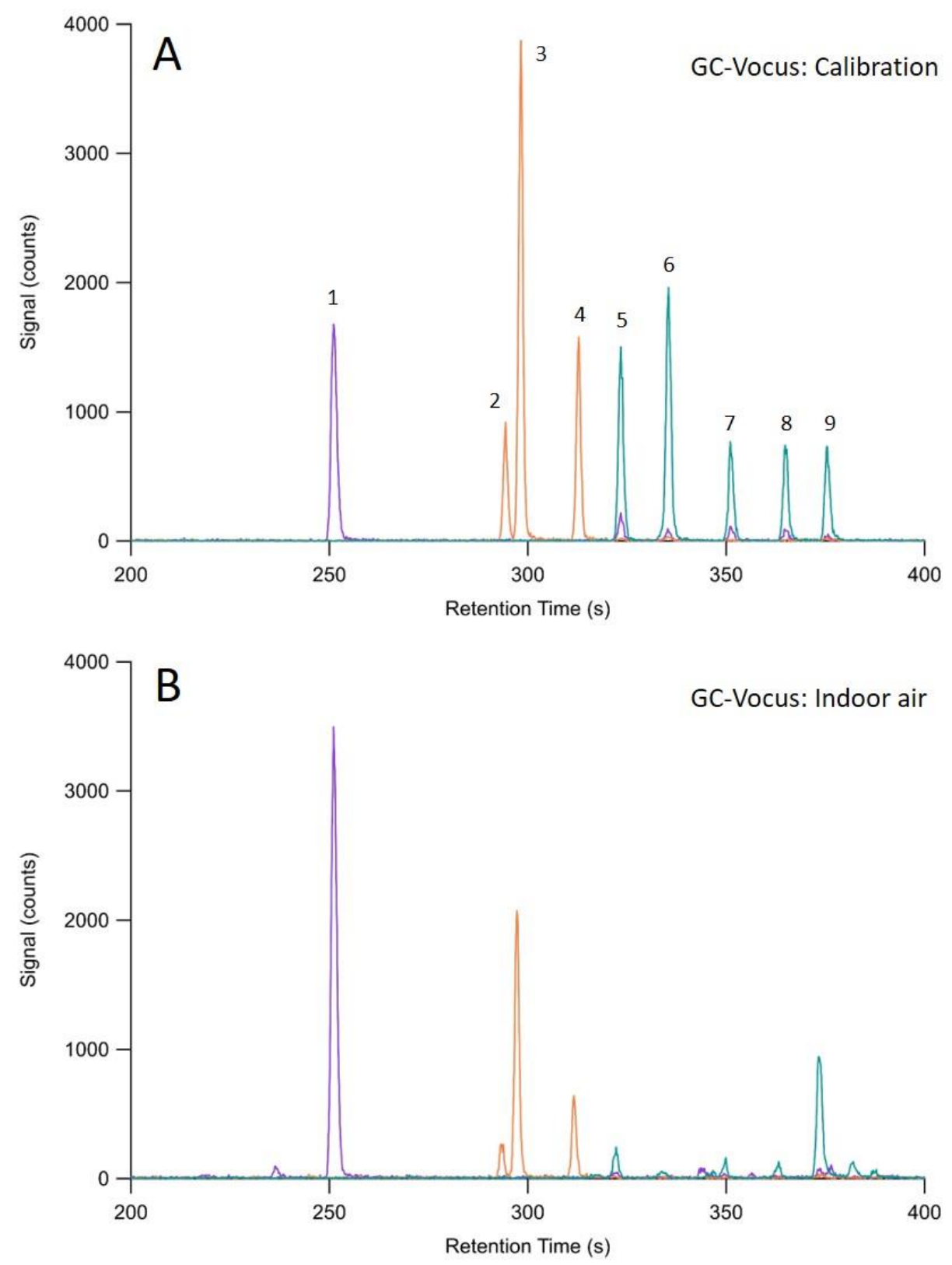

Figure S2. GC-Vocus chromatograms demonstrating the separation of monoterpenes and $\mathrm{C}_{7}$ and $\mathrm{C}_{8}$ aromatics in (A) the calibration mixture and (B) ambient indoor air. The compounds labeled in the calibration chromatogram are (1) toluene (2) ethyl benzene (3) m\&p-xylenes (4) o-xylene (5) $\alpha$-pinene (6) camphene (7) $\beta$-pinene (8) carene and (9) limonene. 

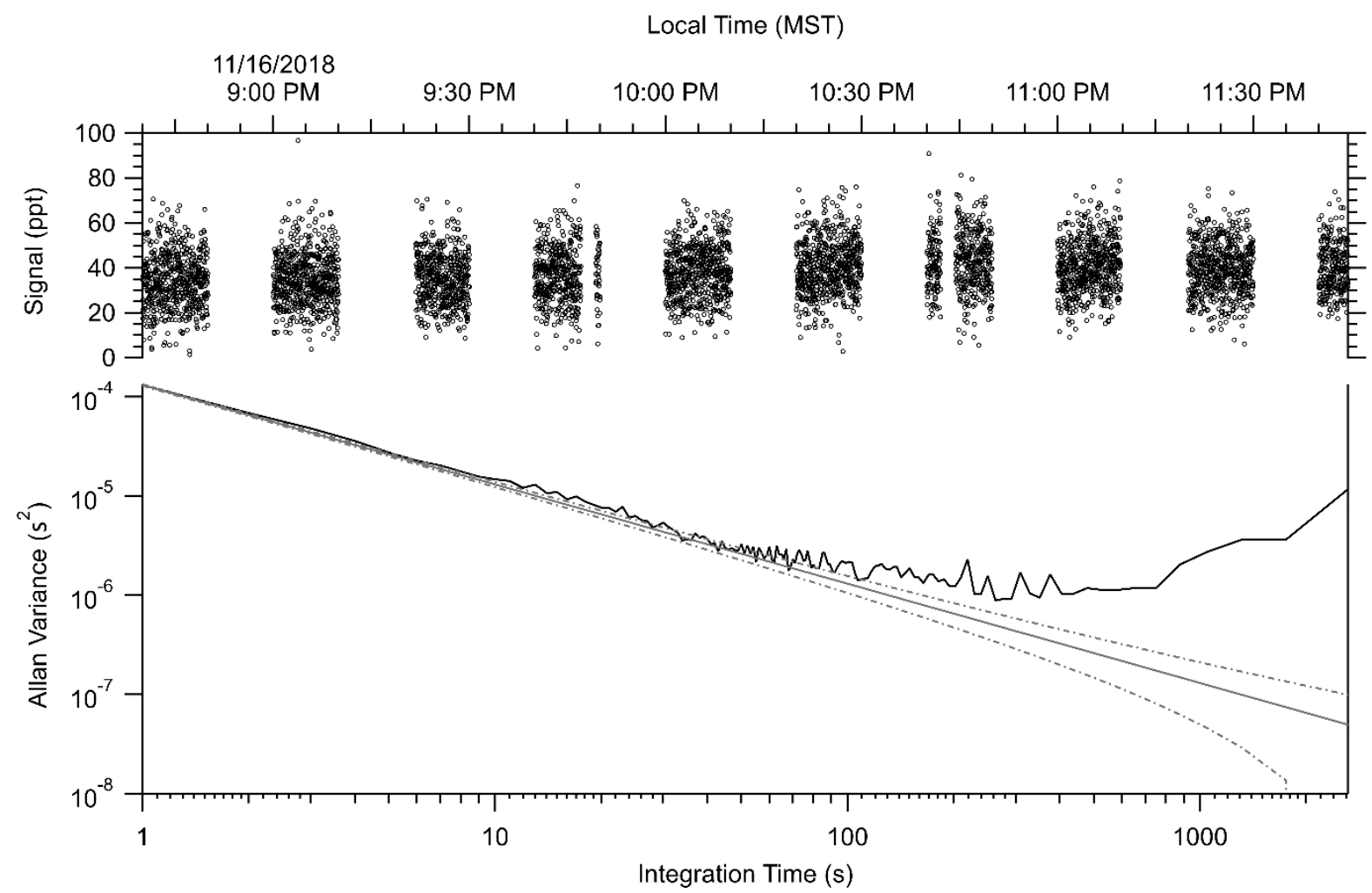

Figure S3. Allan variance plot of the RT-Vocus $\mathrm{C}_{10} \mathrm{H}_{17}{ }^{+}$signal. The RT-Vocus trace contains only room air measurements, periods of sampling from the supply air were removed. The subset of $\mathrm{C}_{10} \mathrm{H}_{17}{ }^{+}$signal was chosen when the concentration was both low and contained minimum perturbations from local sources. The Allan variance analysis results in a minimum at $\sim 250 \mathrm{~s}$ of averaging time, meaning that if the RT-Vocus data was averaged to give one data point every $250 \mathrm{~s}$ the noise could be reduced by actor of $\sim 16$. 


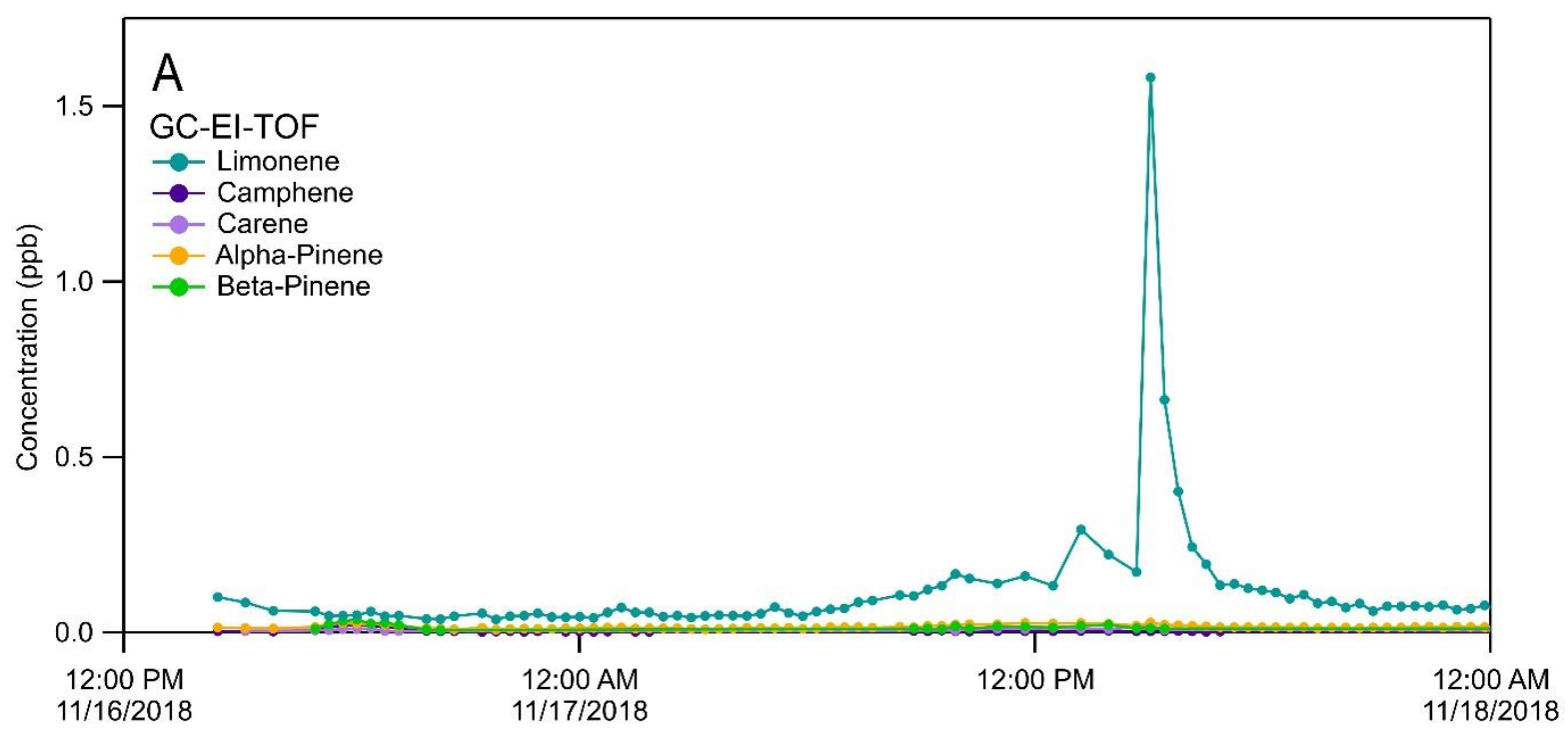

Local Time (MST)

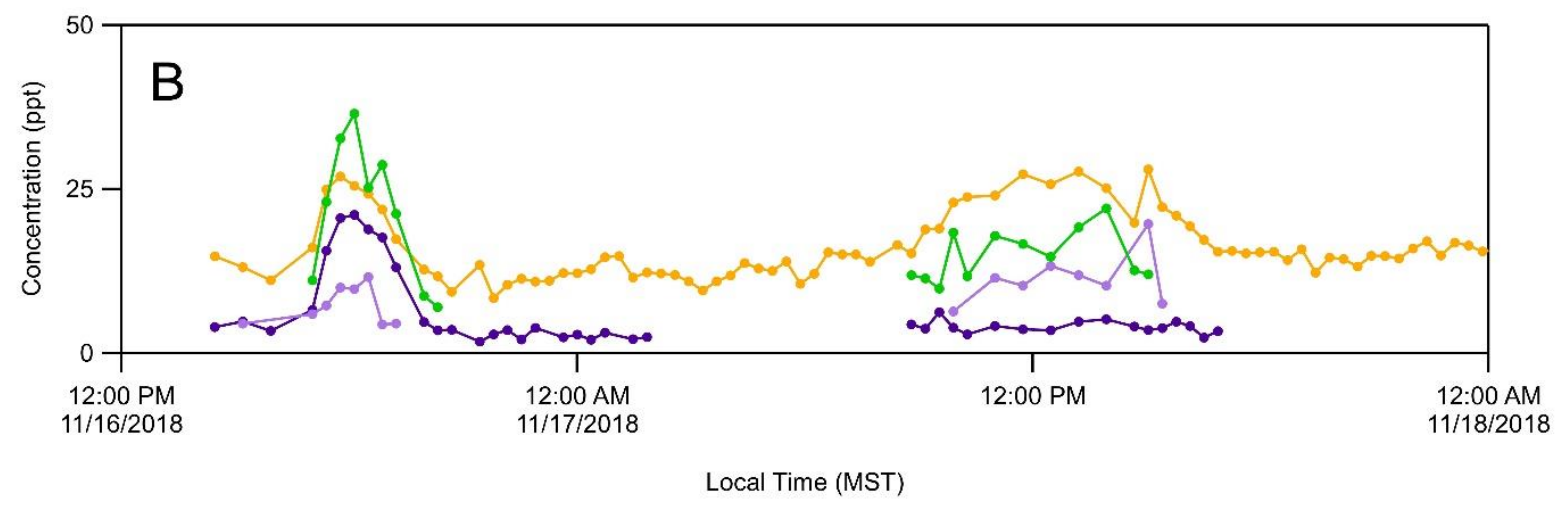

Figure S4. (A) GC-EI-TOF monoterpene time series (B) details of monoterpenes detected in lower ambient concentrations, absent data points are for chromatograms where the chromatographic peak was below the limit of detection. Large peak in limonene on November 17 is due to enhancements observed after the football game, while the increase in signal for the other monoterpenes on November 16 is associated with the inflow of outdoor air into the room. 

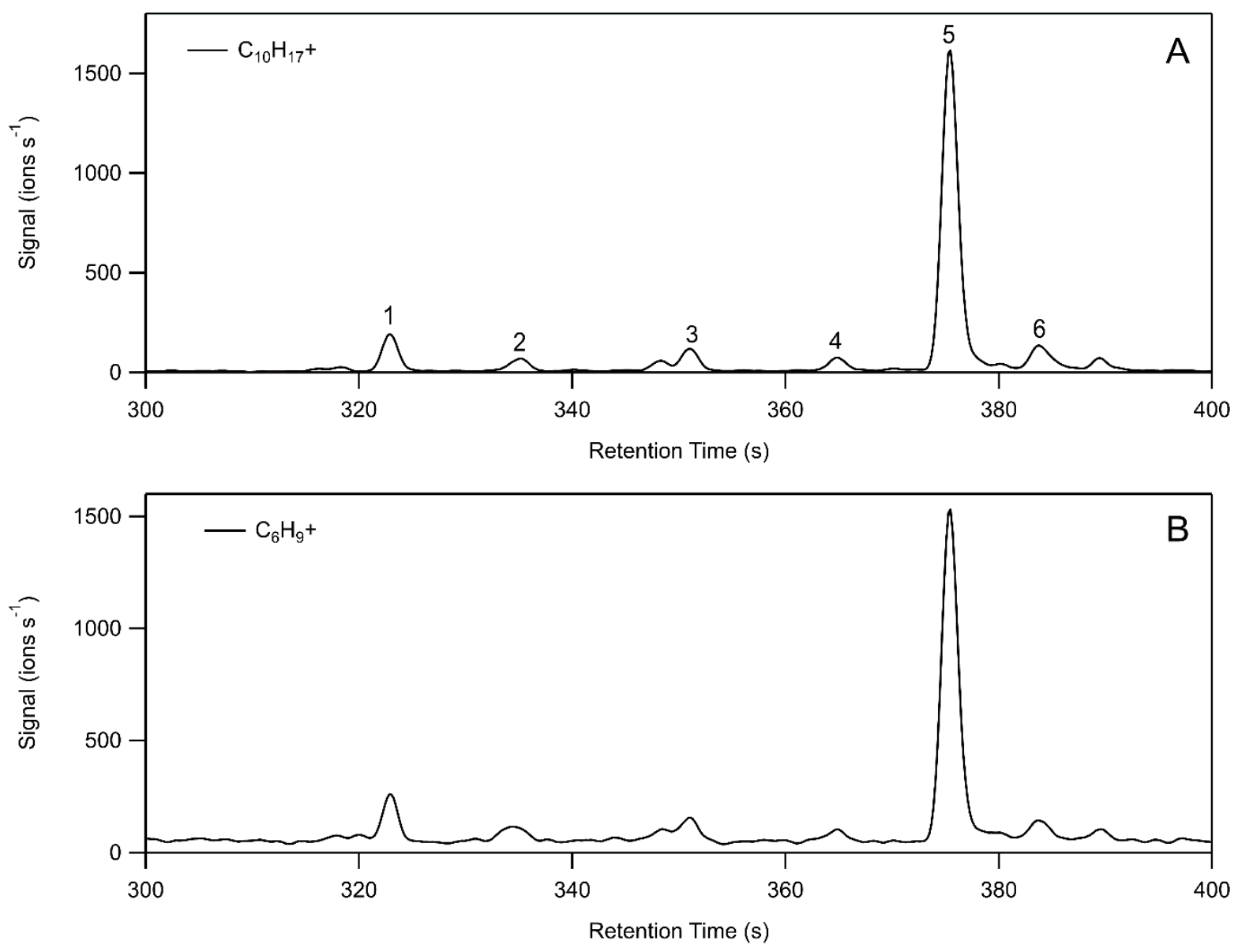

Figure S5. Ambient GC-Vocus chromatogram, showing subsection of the chromatogram where the monoterpenes elute, demonstrating separation of Vocus signal (A) $\mathrm{C}_{10} \mathrm{H}_{17}{ }^{+}(\mathrm{m} / z$ 137.1325), the protonated molecular ion of monoterpenes where peaks labeled $1-6$ correspond to $\alpha$-pinene, camphene, $\beta$-pinene, carene, limonene, and $\gamma$-terpinene, respectively, and (B) $\mathrm{C}_{6} \mathrm{H}_{9}{ }^{+}(\mathrm{m} / z$ 81.0699) ion being detected here as fragmentation ions of the monoterpenes. 

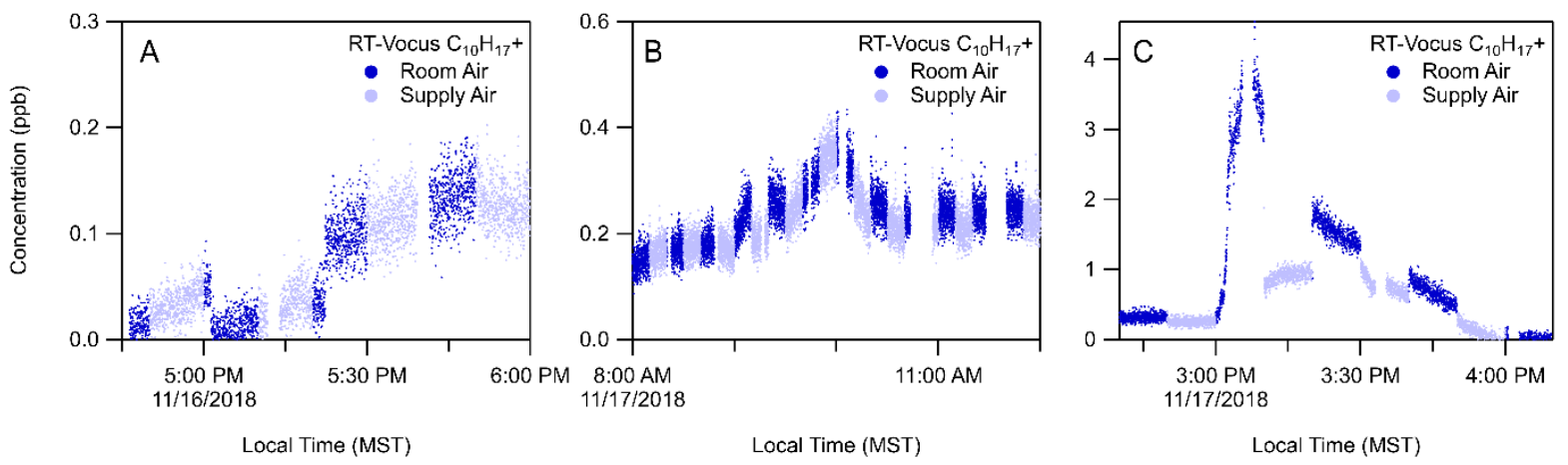

Figure S6. RT-Vocus detection of $\mathrm{C}_{10} \mathrm{H}_{17}{ }^{+}$during A) elevation increase on November 16, initially observed in the supply air indicating an outdoor source of biogenics B) the tailgating and football game event on November 17 where both the room and supply air showed similar elevated signals indicating a source in close proximity to the weight room and C) elevation increase after the football event on November 17, where the increase in signal was initially detected in the room air at $3 \mathrm{pm}$, followed by subsequent increase in the supply air, indicating an in-room source of monoterpene emissions. 

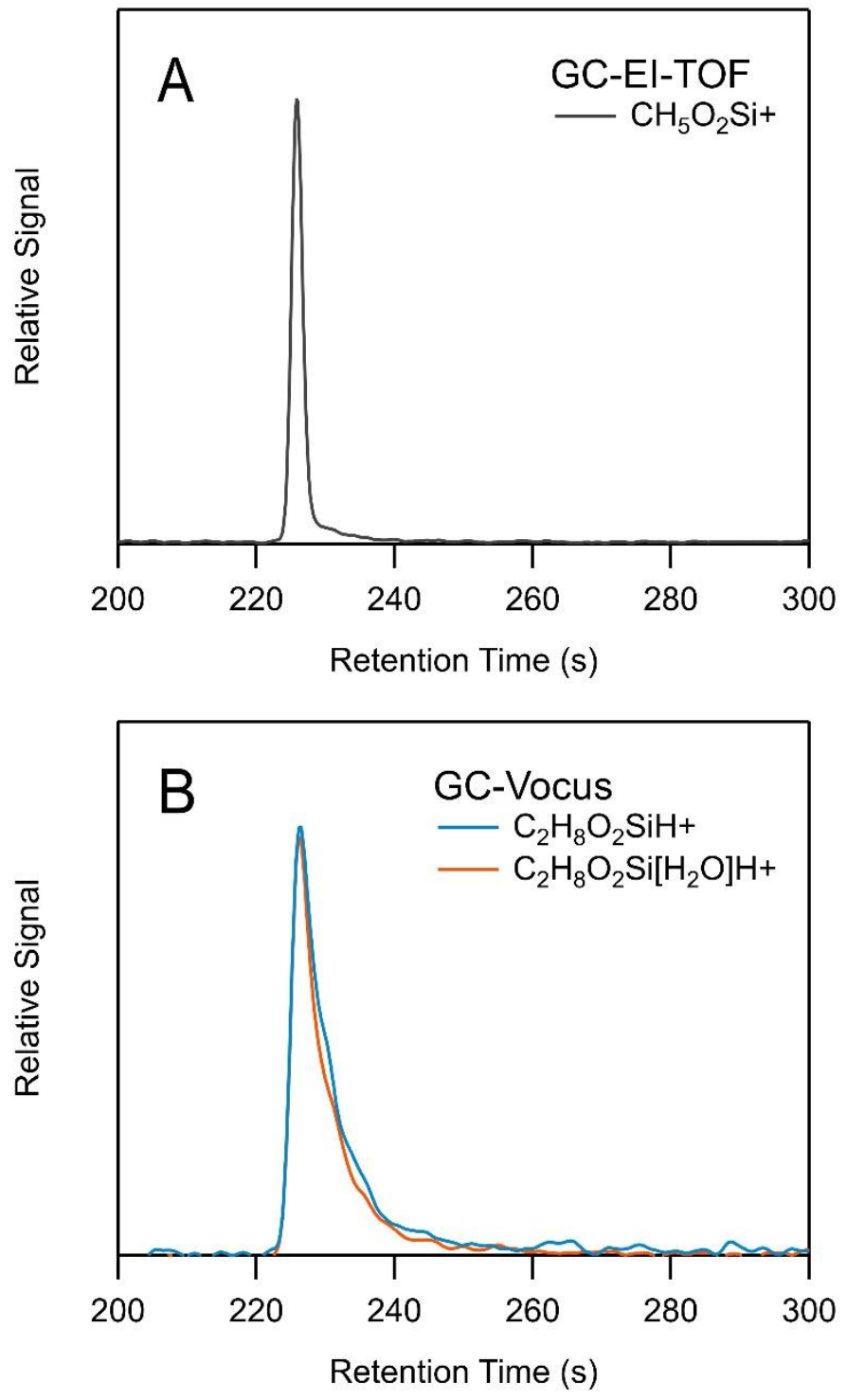

Figure S7. Chromatographic separation of DMSD with retention time $230 \mathrm{~s}$ (A) GC-EI-TOF detection of DMSD characteristic ion $\mathrm{CH}_{5} \mathrm{O}_{2} \mathrm{Si}^{+}$(B) $\mathrm{GC}$-Vocus detection of both the protonated molecular ion, $\mathrm{C}_{2} \mathrm{H}_{8} \mathrm{O}_{2} \mathrm{SiH}^{+}$, and its water cluster, $\mathrm{C}_{2} \mathrm{H}_{8} \mathrm{O}_{2} \mathrm{Si}\left[\mathrm{H}_{2} \mathrm{O}\right] \mathrm{H}^{+}$. 

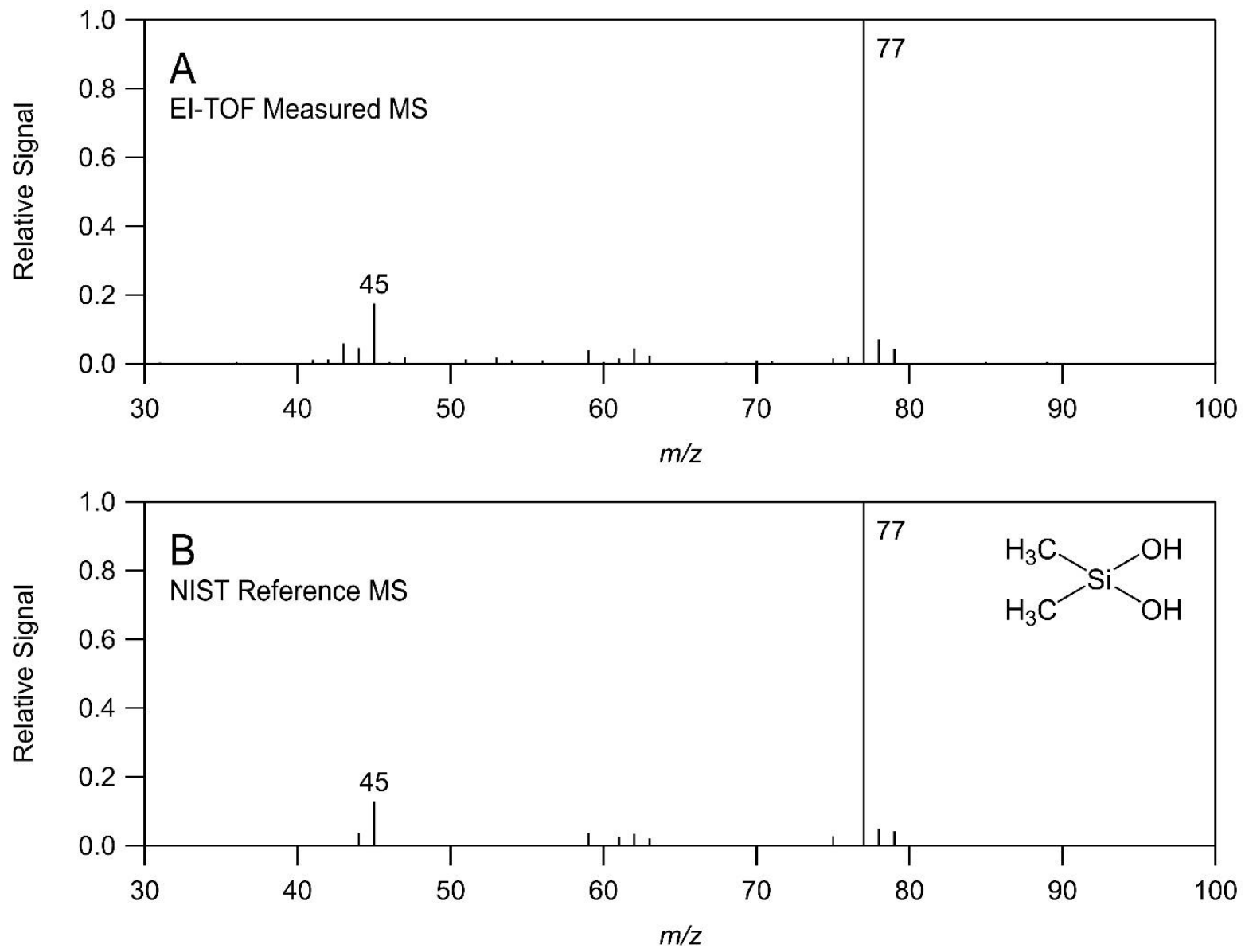

Figure S8. Electron ionization mass spectrum of DMSD for (A) chromatographic peak obtained by GCEI-TOF and (B) standard NIST mass spectrum. 

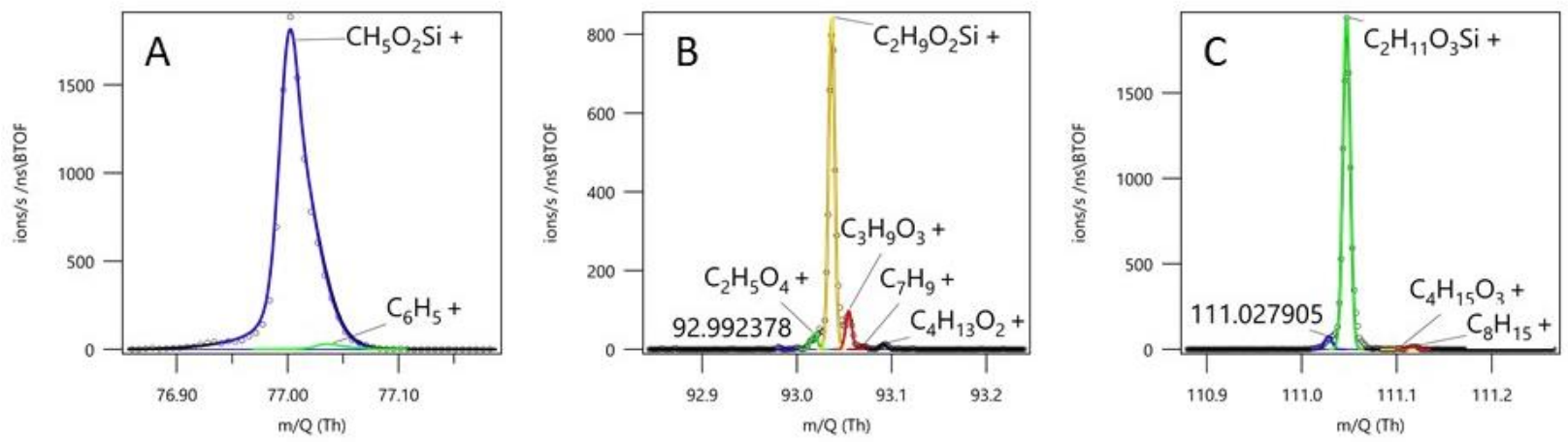

Figure S9. Mass spectrum high resolution peak fits of DMSD during chromatographic separation (A) characteristic fragmentation ion, $\mathrm{CH}_{5} \mathrm{O}_{2} \mathrm{Si}^{+}$, detected by EI-TOF (B) protonated molecular ion, $\mathrm{C}_{2} \mathrm{H}_{8} \mathrm{O}_{2} \mathrm{SiH}^{+}$, and (C) its water cluster, $\mathrm{C}_{2} \mathrm{H}_{8} \mathrm{O}_{2} \mathrm{Si}\left[\mathrm{H}_{2} \mathrm{O}\right] \mathrm{H}^{+}$, detected by Vocus. 


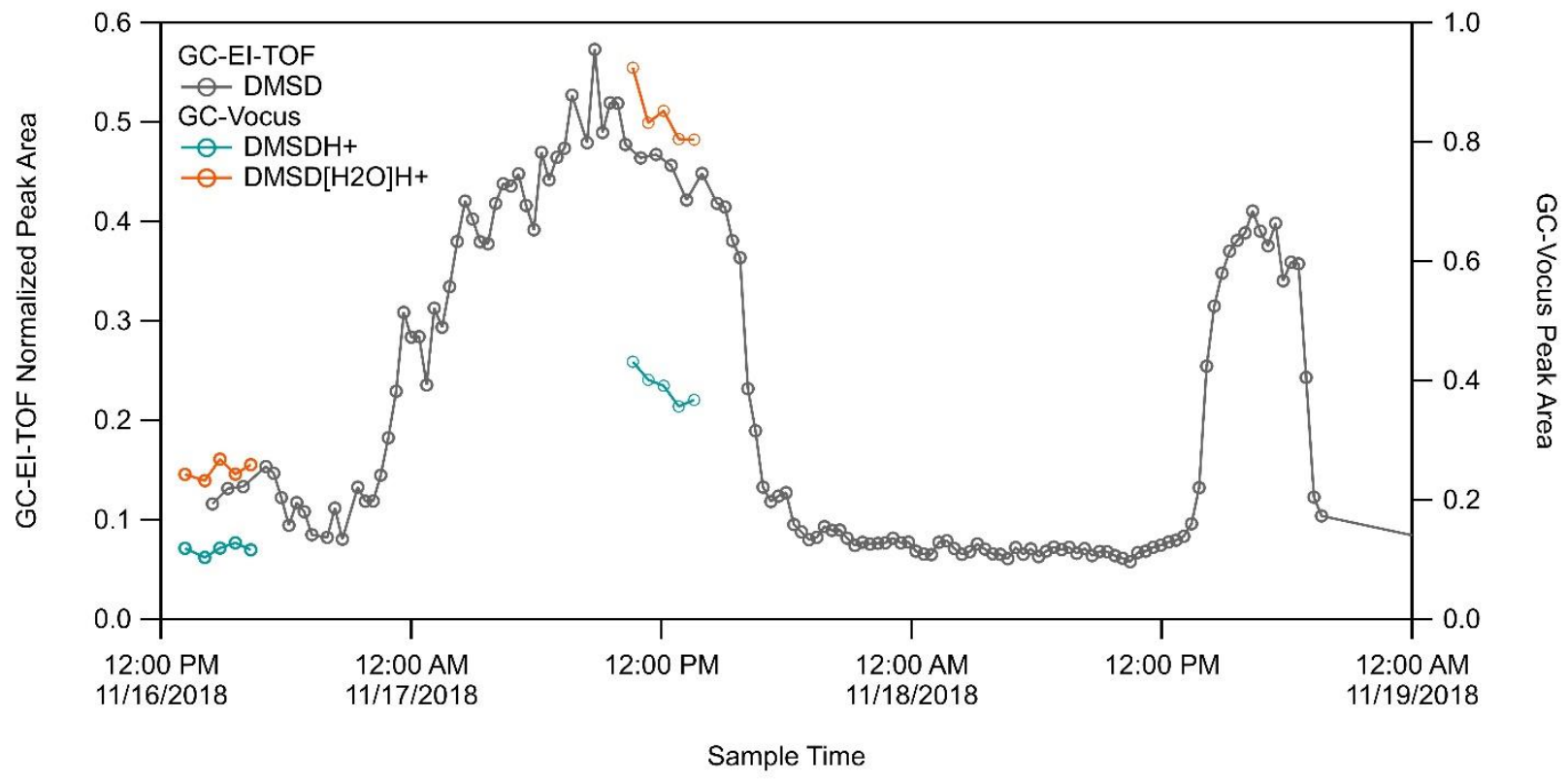

Figure S9. Time series of DMSD detected by GC-EI-TOF (grey trace) and GC-Vocus (blue trace, protonated molecular ion; orange trace, water cluster of protonated molecular ion). 